\title{
Hyperkalemia-Induced Coronary Artery Spasm and Junctional Tachycardia in Diabetic Ketoacidosis Reversed with Insulin and Saline, A Case Report
}

\author{
Yasser Mohammed Hassanain Elsayed* \\ Critical Care Unit, Fraskour Central Hospital, Egypt
}

*Corresponding author: Critical Care Unit, Fraskour Central Hospital, Damietta Health Affairs, Egyptian Ministry of Health (MOH), Damietta, Egypt

\begin{abstract}
Rationale: Serum potassium concentration is usually elevated in the cases of diabetic ketoacidosis. Coronary artery spasm is recognized after the hematological chemical disturbance. Hyperkalemia is a rare cause of junctional tachycardia. Insulin decreases potassium levels in the blood by redistributing it into cells via increased sodium-potassium pump activity.

Patient concerns: A young housewife female patient presented to the emergency department with diabetic ketoacidosis, coronary artery spasm, and junctional tachycardia.

Diagnosis: Hyperkalemia-induced coronary artery spasm and junctional tachycardia in diabetic ketoacidosis.

Interventions: Electrocardiography, oxygenation, central venous pressure monitoring, and echocardiography.

Lessons: Electrolytes disturbance especially hyperkalemia is a significant serious metabolic problem in ketoacidosis. Hyperkalemia is a possible cause for both coronary artery spasm and junctional tachycardia in diabetic ketoacidosis.

Outcomes: Successful reversal of ketotic hyperkalemia-induced coronary artery spasm and junctional tachycardia with insulin and saline.
\end{abstract}

Keywords: Ketotic hyperkalemia; Induced junctional tachycardia; Insulin and saline; Diabetic ketoacidosis

Abbreviations: AVN: Atrioventricular node; CAS: Coronary artery spasm; DKA: Diabetic ketoacidosis; ECG: Electrocardiogram; ICU: Intensive care unit; SAN: Sinoatrial node

\section{Introduction}

The admission serum potassium concentration is usually elevated in patients with diabetic ketoacidosis (DKA) [1]. Potassium levels can fluctuate severely during the treatment of DKA because insulin decreases potassium levels in the blood by redistributing it into cells via increased sodium-potassium pump activity [2]. Despite a total body potassium deficit of $\sim 3-5 \mathrm{mEq} / \mathrm{kg}$ of body weight, most patients with DKA have a serum potassium level at or above the upper limits of normal. In a recent series, the mean serum potassium in patients with DKA was $5.6 \mathrm{mEq} / \mathrm{l}$, respectively. These high levels occur because of a shift of potassium from the intracellular to the extracellular space due to acidemia, insulin

deficiency, and hypertonicity [1]. The primary treatment of DKA is with intravenous fluids and insulin [3]. Both insulin therapy and correction of acidosis decrease serum potassium levels by stimulating cellular potassium uptake in peripheral tissues1. A large part of the shifted extracellular potassium would have been lost in urine because of osmotic diuresis. Hypokalemia (low blood potassium concentration) often follows treatment [2]. Therefore, to prevent hypokalemia, most patients require intravenous potassium during DKA therapy. This increases the risk of dangerous irregularities in the heart rate. Therefore, continuous observation of the heart rate is recommended [2], as well as the repeated 
measurement of the potassium levels and addition of potassium to the intravenous fluids once levels fall below $5.3 \mathrm{mmol} / \mathrm{l}$. If potassium levels fall below $3.3 \mathrm{mmol} / \mathrm{l}$, insulin administration may need to be interrupted to allow correction of the hypokalemia [4]. Replacement with intravenous potassium (two-thirds as potassium chloride $[\mathrm{KCl}]$ and one-third as potassium phosphate [KPO4] should be initiated as soon as the serum potassium concentration is below $5.0 \mathrm{mEq} / \mathrm{L}$. The treatment goal is to maintain serum potassium levels within the normal range of $4-5 \mathrm{mEq} / \mathrm{L}^{1}$.

Coronary artery spasm (CAS) is an intense vasoconstriction of coronary arteries that causes total or subtotal vessel occlusion [5]. CAS or smooth muscle constriction of the coronary artery is an important cause of chest pain syndromes that can lead to myocardial infarction, ventricular arrhythmias, and sudden cardiac death. About $2 \%$ of people with angina, or chest pain and pressure, experience CAS [6]. The attacks of CAS are associated with either STsegment elevation or depression on electrocardiogram (ECG) [7]. The injury to vessels causes spasm are recognized after chemical irritation, physical trauma, or ischemia [8]. Smoking, age and highsensitivity C-reactive protein (hs-CRP) are significant risk factors for CAS. Calcium antagonists are an essential medical therapeutic option. Coronary angiography and provocative testing are the main diagnostic tools [5]. Coronary angiography is the gold standard for the diagnosis of variant angina. Several provocative tests for CAS are used, including ergonovine, acetylcholine, neuropeptide $\mathrm{Y}$ and dopamine [9].

The implicated nomenclature to recognize the type of junctional rhythms (JR) is based on their rate. They are classified as follows:

a. Junctional bradycardia: Ventricular rate $<40 \mathrm{bpm}$.

b. Junction escape rhythm: Ventricular rate 40-60 bpm.

c. Accelerated junctional rhythm (AJR): Ventricular rate of 60-100 bpm.

d. Junctional tachycardia: Ventricular rate $>100 \mathrm{bpm}$ [10].

If there is a blockage for the sinoatrial node (SAN) electrical activity is blocked or is less than the automaticity of the atrioventricular node $(\mathrm{AVN}) / \mathrm{His}$ Bundle a JR starts. Numerous conditions and medications can lead to a diseased SAN and lead to the AVN/His Bundle to take over due to the higher automaticity of the ectopic pacemaker [11-13]. Hyperkalemia, sick sinus syndrome, pericarditis, myocarditis, unstable angina, acute myocardial infarction, repair of congenital heart disease, atrial septal defect, tetralogy of Fallot, persistent left superior vena cava, adenosine, digoxin, calcium channel blockers, lithium, amitriptyline, clonidine, reserpine, inhalation anesthetics, cimetidine, isoproterenol infusion, narcotics, beta-blockers, and ivabradine implicated in causing junctional tachycardia [10,14-17].

\section{Case Presentation}

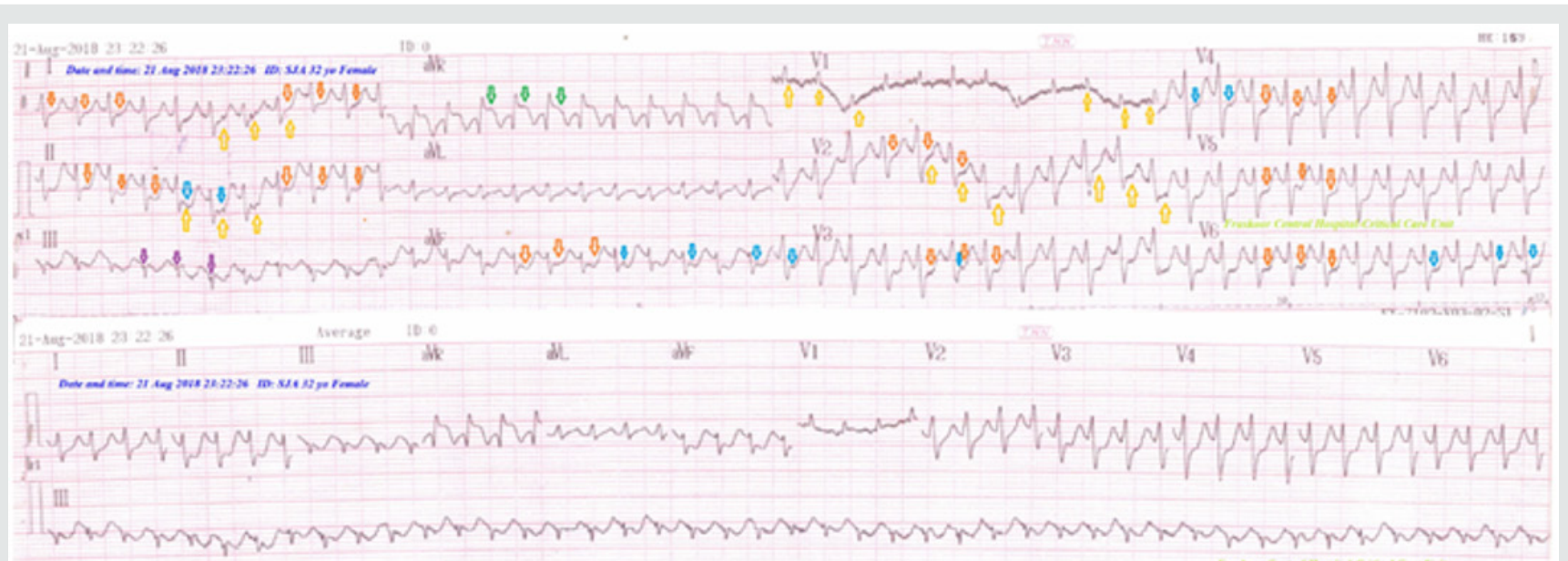

Figure 1: ECG tracings showing junctional tachycardia; "evidenced with inverted P-wave (purple arrows), retrograde P-wave (blue arrows), and absent P-wave (the remaining leads)" extensive straight ST-segment depressions in infero-anterior ECG leads (II, aVF, I, and V2-6) (red arrows), ST-segment elevation in aVR lead (green arrows), and wavy triple ECG sign of hypocalcemia in leads (gold arrows).

A 32-year-old housewife Egyptian female patient presented to the emergency department with chest pain, palpitations, and rapid breathing. The patient had a history of diabetics on longacting insulin. She gave a history of missed insulin for 3 days. Upon examination, the patient appeared confused, sweaty, and tachypneic. His vital signs were as follows: the blood pressure of $90 / 70 \mathrm{mmHg}$, the heart rate of $150 /$ minute, the temperature of $36.9^{\circ} \mathrm{C}$, respiratory rate of $34 / \mathrm{min}$, and the initial pulse oximetry of $94 \%$. The patient was admitted to the intensive care unit (ICU) as diabetic ketoacidosis. The patient initially treated with act-rapid insulin (initial 8 units IVB), normal saline 0.9\% (1000 ml IVB in the first hour), hourly vital signs monitoring, hourly blood glucose measurement, and hourly urinary acetone check-up. Maintenance of act-rapid insulin $(0.1 \mathrm{u} / \mathrm{kg} ; 8 \mathrm{u} /$ hours $)$ was continued for about 6 hours. Another 1000 ML normal saline $0.9 \%$ was added. RBS was $568 \mathrm{mg} / \mathrm{dl}$ on admission and urine analysis (glucose ++++ acetone +++ ). ABG showed metabolic acidosis. Electrolytes profile showed plasma $\mathrm{Na}^{+}: 131 \mathrm{mg} / \mathrm{dl}$, plasma $\mathrm{Ka}^{+}: 6.1 \mathrm{mg} / \mathrm{dl}, \mathrm{I} \mathrm{Ca}^{++}: 0.65 \mathrm{mmol} / \mathrm{L}$. Urgent ECG recording showed junctional tachycardia, extensive STsegment depressions and wavy ECG sign of hypocalcemia (Figure 
1). Serial ECG tracings were done for follow up. RBS measures were steadily gradually decreased until RBS become;174 mg/dl, the disappearance of urinary acetone. IVI dextrose $5 \%$ added with adjustment of act-rapid insulin to (4 units per hour), and A $250 \mathrm{ml}$ Ringer solution over 2 hours, and $250 \mathrm{ml}$ Ringer solution over 2 hours. Electrolytes profile repeated within 6 hours of management showed normalized plasma $\mathrm{Na}^{+}: 141 \mathrm{mg} / \mathrm{dl}$, plasma $\mathrm{Ka}^{+}: 5.2 \mathrm{mg} /$ dl, $\mathrm{I} \mathrm{Ca}^{++}: 1.1 \mathrm{mmol} / \mathrm{L}$. ECG tracing on discharge was showed the disappearance of junctional tachycardia with normalization of the above ST-segment depressions and wavy ECG sign of hypocalcemia (Figure 2). Later echocardiography was normal. The patient discharged after stability on the fourth day of admission.

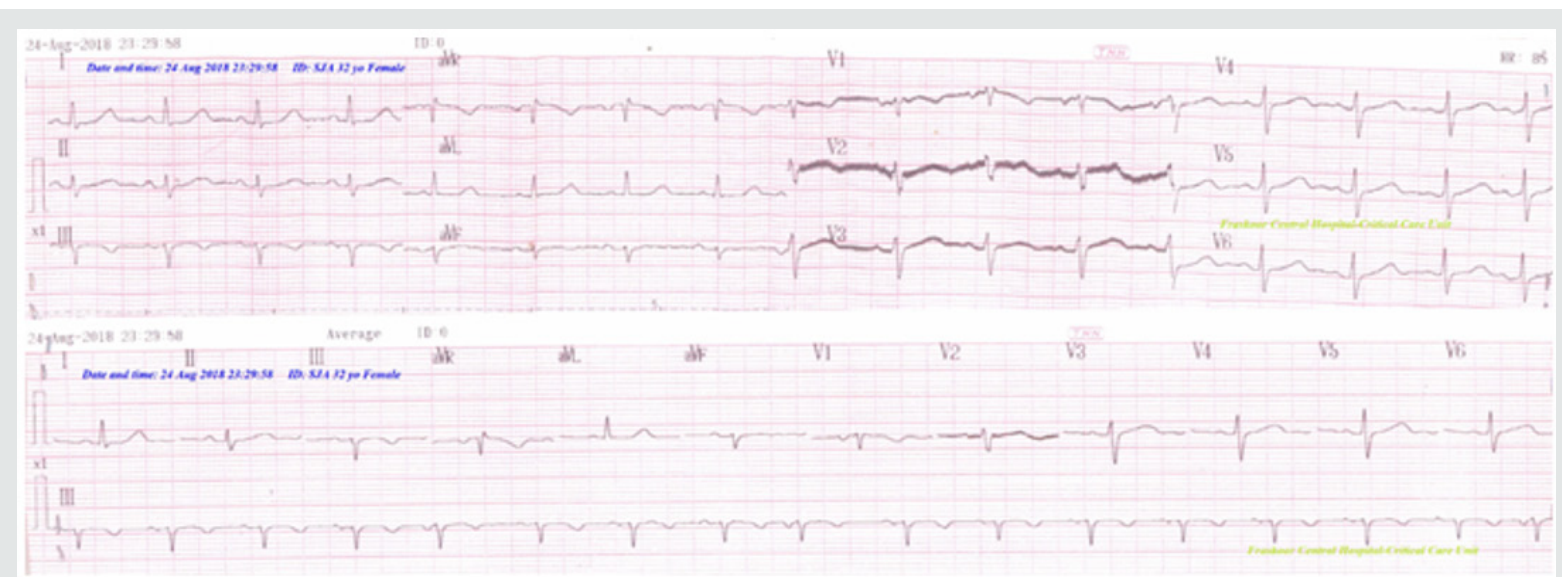

Figure 2: ECG tracings showing the disappearance of junctional tachycardia with normalization of the above ST-segment depressions and wavy triple ECG sign of hypocalcemia.

\section{Discussion}

Overview: A 32-year-old single Egyptian male patient presented with diabetic ketoacidosis, junctional tachycardia, and coronary artery spasm in the presence of marked hyperkalemia.

The primary objective: for my case study was the diagnosis of diabetic ketoacidosis, junctional tachycardia, and coronary artery spasm.

The secondary objective: for my case study was the choice management for diabetic ketoacidosis, junctional tachycardia, and coronary artery spasm in the presence of marked hyperkalemia.

Limitations of the study: There are no known limitations in the study.

I can't compare the current case with similar conditions: There are no similar or known cases with the same management for near comparison.

Study question here: How did the relationship among diabetic ketoacidosis and junctional tachycardia, elevated potassium level, coronary artery spasm, and insulin therapy?

\section{Recommendations}

a. It is recommended to widening the research in clearing the effect of diabetic ketoacidosis on potassium elevation and its role in causing junctional tachycardia and coronary artery spasm.

b. Also, it is recommended to extend the research on the impact of rapid-acting insulin therapy in the management of diabetic ketoacidosis on junctional tachycardia, coronary artery spasm, and elevated potassium level.

\section{Conclusion}

The physician should consider spontaneous recovery of junctional tachycardia, coronary artery spasm, and hyperkalemia on the initial management of diabetic ketoacidosis. So, don't hurry to treatment, but tight follow up for elevated potassium level is obligatory.

\section{Conflicts of Interest}

There are no conflicts of interest.

\section{Acknowledgment}

I wish to thank the emergency department and critical care unit nurses who make extra-ECG copies for helping me.

\section{References}

1. Umpierrez GE, Murphy MB, Kitabchi AE (2002) Diabetic Ketoacidosis and Hyperglycemic Hyperosmolar Syndrome. Diabetes Spectrum 15(1): 28-36.

2. Edge J (2009) BSPED Recommended DKA Guidelines 2009. British Society for Paediatric Endocrinology and Diabetes.

3. Misra S, Oliver NS (2015) Diabetic ketoacidosis in adults. BMJ (Clinical Research edn), 28(351): h5660.

4. Kitabchi AE, Umpierrez GE, Miles JM, Fisher JN (2009) Hyperglycemic crises in adult patients with diabetes. Diabetes Care 32(7): 1335-1343.

5. Hung MJ, Hu P, Hung MY (2014) Coronary Artery Spasm: Review and Update. Int J Med Sci 11(11): 1161-1171.

6. Moore K (2017) Coronary Artery Spasm. Healthline 2017. Copyright (C) 2005 - 2018 Healthline Media.

7. Yasue H, Nakagawa H, Itoh T, Harada E, Mizuno Y (2008) Coronary artery spasm-Clinical features, diagnosis, pathogenesis, and treatment. Journal of Cardiology 51: 2-17. 
8. Hellstrom HR (1975) The advantages of a vasospastic cause of myocardial infarction. AHJ 5: 545-548.

9. Zaya M, Mehta PK, Merz CN (2014) Provocative testing for coronary reactivity and spasm. Am Coll Cardiol 63(2): 103-109.

10. Hafeez Y, Grossman SA (2019) Junctional Rhythm. StatPearls Publishing LLC.

11. Semelka M, Gera J, Usman S (2013) Sick sinus syndrome: a review. Am Fam Physician. 87(10): 691-696.

12. Trappe HJ (2010) Tachyarrhythmias, bradyarrhythmias and acute coronary syndromes. J Emerg Trauma Shock 2: 137-142.

13. Romhilt DW, Doyle M, Sagar KB, Hastillo A, Wolfgang TC, et al. (1982) Prevalence and significance of arrhythmias in long-term survivors of cardiac transplantation. Circulation 62(2): I219-222.
14. Arı H, Kahraman F, Baș HA, Arslan A (2015) Low atrial rhythm mimics myocardial infarction. Anatol J Cardiol 15(8): 675-683.

15. Beinart SC (2017) Junctional Rhythm. Medscape.

16. Elsayed YMH (2020) A Case Report in Cardiology, Emergency and Critical Care Ketotic Hyperkalemia-Induced Junctional Tachycardia Reversed with Insulin and Saline; A Case Report. Emergency Medicine and Trauma Care Journal.

17. Kishimoto N, Kinoshita I, Momota Y (2017) Junctional Rhythm Preoperatively and During General Anesthesia for Oral and Maxillofacial Surgery. Anesth Prog 64(3): 165-167.

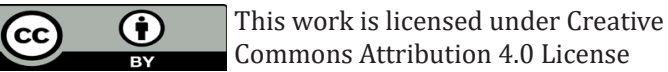

To Submit Your Article Click Here:

Submit Article

DOI: $10.32474 /$ GJAPM.2020.02.000149

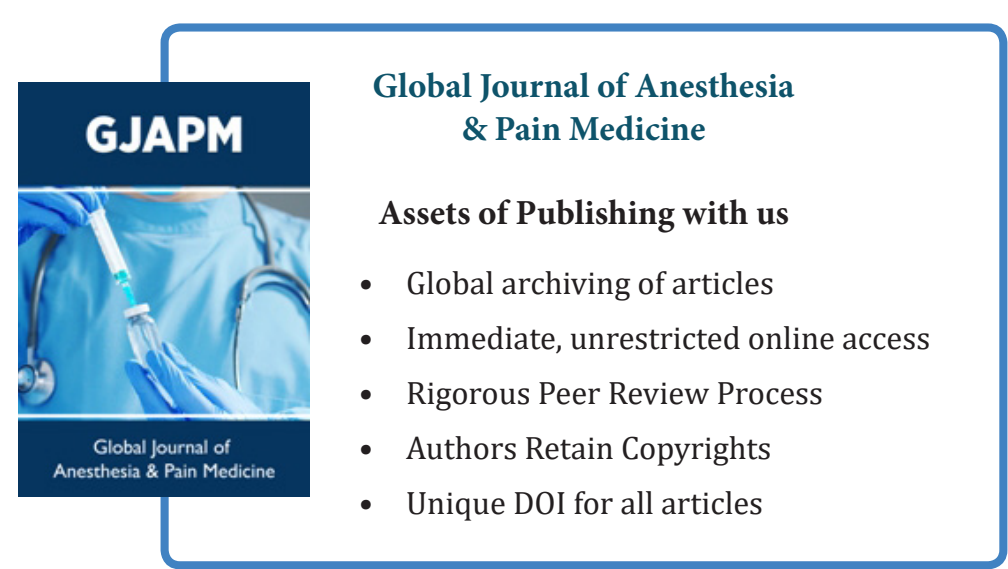

